\title{
Nonlinear Model Predictive Control using Feedback Linearization and Local Inner Convex Constraint Approximations
}

\author{
Daniel Simon, Johan Löfberg and Torkel Glad
}

\section{Linköping University Post Print}

\section{Tweet}

N.B.: When citing this work, cite the original article.

Original Publication:

Daniel Simon, Johan Löfberg and Torkel Glad, Nonlinear Model Predictive Control using Feedback Linearization and Local Inner Convex Constraint Approximations, 2013, Proceedings of the 2013 European Control Conference, 2056-2061.

From the 2013 European Control Conference, July 17-19, Zurich, Switzerland.

Postprint available at: Linköping University Electronic Press http://urn.kb.se/resolve?urn=urn:nbn:se:liu:diva-96743 


\title{
Nonlinear Model Predictive Control using Feedback Linearization and Local Inner Convex Constraint Approximations
}

\author{
Daniel Simon Johan Löfberg Torkel Glad \\ Departement of Electrical Engineering \\ Linköping University \\ SE-581 83 Linköping, Sweden
}

\begin{abstract}
Model predictive control (MPC) is one of the most popular advanced control techniques and is used widely in industry. The main drawback with MPC is that it is fairly computationally expensive and this has so far limited its practical use for nonlinear systems.

To reduce the computational burden of nonlinear MPC, Feedback Linearization together with linear MPC has been used successfully to control nonlinear systems. The main drawback is that this results in an optimization problem with nonlinear constraints on the control signal.

In this paper we propose a method to handle the nonlinear constraints that arises using a set of dynamically generated local inner polytopic approximations. The main benefits of the proposed method is guaranteed recursive feasibility and convergence.
\end{abstract}

\section{INTRODUCTION}

Model Predictive Control is an optimal control strategy where instead of solving an infinite horizon optimal control problem for the discrete-time system

$$
x_{k+1}=f\left(x_{k}, u_{k}\right)
$$

one tries to approximate the solution with a finite horizon optimal control problem

$$
\begin{aligned}
\min _{u} & \Psi\left(x_{k+N}\right)+\sum_{i=0}^{N-1} \ell\left(x_{k+i}, u_{k+i}\right) \\
\text { s.t. } & \\
x_{k+i+1}= & f\left(x_{k+i}, u_{k+i}\right) \\
x_{k+i} & \in \mathcal{X} \\
u_{k+i} & \in \mathcal{U} \\
x_{k+N} & \in \mathcal{T}
\end{aligned}
$$

The finite time optimization problem is solved in each sample time $k$ for a horizon of $N$ time samples ahead [1]. The result of this is an open-loop control sequence $u_{k: k+N-1}=\left[u_{k}, u_{k+1}, \ldots, u_{k+N-1}\right]$. The first sample, $u_{k}$, from the control sequence is then applied to the system, and the whole procedure is repeated in the next time instance $k+1$, yielding a so called receding horizon control. This gives in practice a closed loop feedback optimal control strategy [2].

Among the many different formulations of this finite time optimal control problem the one that has attracted a lot of attention and is widely used is the formulation with a terminal cost and constraint set [1]. In practice this approach adds the final state cost term $\Psi\left(x_{k+N}\right)$ and a constraint $\mathcal{T}$ on the final state $x_{N}$ to the optimization problem. The constraint $\mathcal{T} \subseteq \mathcal{X}$ is normally chosen to be a positively invariant set [3] of the system under the feedback $u_{k}=\kappa\left(x_{k}\right) \in \mathcal{U}$ and $\Psi\left(x_{k+N}\right)$ is chosen to be a Lyapunov function associated with local controller $u_{k}=\kappa\left(x_{k}\right)$, since this guarantees, under conditions relating $\Psi$ and $\ell$ that the closed loop system is stable and asymptotically converges to the origin [1].

If either the system (1) is nonlinear or the cost function (2) is a nonconvex function of the states and controls the resulting optimization problem becomes a Nonconvex Program which is, in general, much harder to solve than the Quadratic program that a linear system and quadratic cost function results in [4]. The survey [5] of Cannon reviews several different techniques to solve the nonlinear MPC problem using, e.g., Sequential Quadratic Programming, Euler-Lagrange and Hamilton-Jacobi-Bellman approaches and Cost and constraint approximation.

Yet another way to handle nonlinear systems in MPC without having to globally solve the complicated nonconvex optimization problem is to first create a linear response from input to output of the system [6]. This can be accomplished with inner loop feedback linearization [7] of the form

$$
u=\gamma(x, \tilde{u})
$$

giving a closed loop system which is linear from $\tilde{u}$ to $y$

$$
\dot{z}=A z+B \tilde{u}, \quad y=C z
$$

In section I-A the details of computing $\gamma(x, \tilde{u})$ are outlined.

The first main issues with this is that even if the original cost function (2) is convex the resulting cost function expressed in $\tilde{u}$ can possibly be a nonconvex function. One could simply ignore this complication and formulate a new cost function which is quadratic in $\tilde{u}$. The performance tradeoff is analyzed in [8] with the conclusion that this approximation is justified only when the complete problem then can be formulated as a QP.

The second issue, and in our view a much more problematic issue, is that even simple control signal constraints as

$$
\underline{u} \leq u_{k+i} \leq \bar{u}
$$

will transform into a nonlinear constraints on $\tilde{u}$ using (3).

$$
\pi\left(x_{k+i}, \underline{u}\right) \leq \tilde{u}_{k+i} \leq \pi\left(x_{k+i}, \bar{u}\right)
$$


Several different methods to handle this has been presented in the literature. In e.g., [9] the authors calculate the exact input constraints at time $k$ and use them as constraints on the whole prediction horizon, which clearly does not guarantee recursive feasibility. Other authors such as [10]-[13] propose to use the solution sequence from the previous time step to construct an approximation of the nonlinear constraints. These methods can in general guarantee stability under some strict assumptions, e.g., recursive feasibility. Despite this they can be quite computationally expensive if they work in an iterative manner, e.g., in [10] this approximation is done by iteratively solving the linear MPC problem and in each iteration use the previous solution sequence $x_{k: k+N}^{*}$ to calculate the input constraints using (6). The iterations are cancelled when the solution sequence $u_{k: k+N-1}^{*}$ has converged.

In this paper we will adopt a different approach to handle the nonlinear constraints based on using the exact constraints for the current time step and a set of inner polytope approximations for future time steps.

\section{A. Feedback Linearization}

Let us, before we outline the proposed controller, describe the feedback linearization scheme.

If we consider the affine-in-control nonlinear system of the form

$$
\dot{x}=f(x)+g(x) u, \quad y=h(x)
$$

and define the Lie derivative in the direction of $f$ as

$$
L_{f}=\sum_{i=1}^{n} f_{i}(x) \frac{\partial}{\partial x_{i}}
$$

then, if we repeatedly differentiate the output, we obtain $\dot{y}=$ $L_{f} h, \ddot{y}=L_{f}\left(L_{f} h\right)=L_{f}^{2} h$ etc.

If we assume that the system has $\operatorname{dim} y=\operatorname{dim} u=m$ and apply the Lie derivative then we obtain for the i:th output

$$
\begin{aligned}
\dot{y}_{i}= & L_{(f+g u)} h_{i}=L_{\left.f+u_{1} g_{1}+u_{2} g_{2}+\ldots+u_{m} g_{m}\right)} h_{i} \\
& =L_{f} h_{i}+u_{1} L_{g_{1}} h_{i}+u_{2} L g_{2} h_{i}+\ldots+u_{m} L_{g_{m}} h_{i}
\end{aligned}
$$

Either all $L_{g_{j}} h_{i}=0$ which means that $\dot{y}_{i}=L_{f} h_{i}$ and then we have to keep differentiating in order for $u_{j}$ to affect $y_{i}$, or one of $L_{g_{j}} h_{i} \neq 0$ and then $y_{i}$ is affected by $u_{j}$.

Differentiating one more time gives

$$
\ddot{y}_{i}=L_{f}^{2} h_{i}+u_{1} L_{g_{1}} L_{f} h_{i}+\ldots+u_{m} L_{g_{m}} L_{f} h_{i}
$$

and then if one of the $L_{g_{j}} L_{f} h_{i} \neq 0 u_{j}$ affect $y$ and we say that the system has a relative degree of 2 in $x_{0}$ [7].

This procedure can be summarized in a decoupling matrix $R(x)$ according to

$$
R(x)=\left[\begin{array}{ccc}
L_{g_{1}} L_{f}^{\nu_{1}-1} h_{i} & \ldots & L_{g_{m}} L_{f}^{\nu_{1}-1} h_{i} \\
\vdots & & \vdots \\
L_{g_{1}} L_{f}^{\nu_{m}-1} h_{m} & \ldots & L_{g_{m}} L_{f}^{\nu_{m}-1} h_{m}
\end{array}\right]
$$

which gives

$$
\left[\begin{array}{c}
y_{1}^{\left(\nu_{1}\right)} \\
\vdots \\
y_{m}^{\left(\nu_{m}\right)}
\end{array}\right]=R(x)\left[\begin{array}{c}
u_{1} \\
\vdots \\
u_{m}
\end{array}\right]+\left[\begin{array}{c}
L_{f}^{\nu_{1}} h_{1} \\
\vdots \\
L_{f}^{\nu_{m}} h_{m}
\end{array}\right]
$$

If $R(x)$ is nonsingular then the control signal can be chosen as

$$
u=R^{-1}(x)\left(-\left[\begin{array}{c}
L_{f}^{\nu_{1}} h_{1} \\
\vdots \\
L_{f}^{\nu_{m}} h_{m}
\end{array}\right]+\tilde{u}\right)
$$

and the resulting closed loop system will be linear and decoupled from $\tilde{u}$ to $y$ [7].

This procedure works well when the zero dynamics are stable or when there are no zero dynamics, i.e., when the relative degree is equal to the state dimension. In these cases the system does not need to be transformed into a controllable canonical form [7] and the original system states can be kept which is especially good if we have constraints on the states.

In the rest of this paper we restrict our discussion to these kinds of systems that allow us to keep our original states. The aircraft example in section III-B motivates this choice.

\section{The Proposed Algorithm}

First we make the following assumptions.

Assumption 1: The nonlinear system (7) is input-output feedback linearizable using the control (9) and the linearized system has a discrete-time state-space description

$$
x_{k+1}=A x_{k}+B \tilde{u}_{k}
$$

with no unstable zero dynamics.

Assumption 2: The functions $\Psi(\cdot)$ and $\ell(\cdot)$ are such that they satisfy the necessary conditions for stability [1], the sets $\mathcal{X}$ and $\mathcal{U}$ are convex polytopes and for simplicity we assume $\mathcal{U}$ to be simple bounds on the control signal.

Applying feedback linearization and MPC as described in the previous section to a nonlinear system of the form (7) the resulting MPC problem setup is

$$
\begin{aligned}
\min _{u} & \Psi\left(x_{k+N}\right)+\sum_{i=0}^{N-1} \ell\left(x_{k+i}, \tilde{u}_{k+i}\right) \\
\text { s.t. } & \\
x_{k+i+1}= & A x_{k+i}+B \tilde{u}_{k+i} \\
x_{k+i} & \in \mathcal{X} \\
x_{k+N} & \in \mathcal{T} \\
\tilde{u}_{k+i} & \in \Pi
\end{aligned}
$$

where we have defined

$$
\Pi=\left\{\tilde{u}_{k+i} \mid \pi\left(x_{k+i}, \underline{u}\right) \leq \tilde{u}_{k+i} \leq \pi\left(x_{k+i}, \bar{u}\right)\right\}
$$

where the functions $\pi(\cdot)$ are the nonlinear constraints that arise from feedback linearization (9). 


\section{A. Nonlinear Constraint Approximations}

A first step to handle the nonlinear constraint (11e) is to simply replace it with an global inner convex polytopic approximation

$$
\mathcal{G}=\left\{(x, \tilde{u}) \mid x \in \mathcal{X}, g_{l}(\mathcal{X}) \leq \tilde{u} \leq g_{u}(\mathcal{X})\right\}
$$

where $g_{u}(\mathcal{X})$ is a concave piecewise affine function such that $g_{u}(\mathcal{X}) \leq \pi(\mathcal{X}, \bar{u})$ and $g_{l}(\mathcal{X})$ is a convex piecewise affine function such that $g_{l}(\mathcal{X}) \geq \pi(\mathcal{X}, \underline{u})$. An example of an inner approximation, $\mathcal{G}$, is shown in Figure 1.

Note that this approximation is not unique and the degree of suboptimality vary with the method of approximation.

If the nonlinear constraints (11e) form a highly nonconvex set, then $\mathcal{G}$ is a poor approximation and can only be close to the true constraints in some, possibly small, regions, cutting of control authority in other regions.

This motivates us to not use a global approximation for all time steps in the control signal sequence. To begin with, since the current state is known, the exact nonlinear constraint on $\tilde{u}_{k}$ can be calculated as

$$
\pi\left(x_{k}, \underline{u}\right) \leq \tilde{u}_{k} \leq \pi\left(x_{k}, \bar{u}\right)
$$

Obviously, since this is a linear constraint in $\tilde{u}_{k}$, our scheme should be able to use this exactly without resorting to any conservative approximation. It is thus our goal to derive an algorithm where this constraint is used exactly, and future constraints are included in an as non-conservative fashion as possible, while guaranteeing stability and feasibility.

If one makes use of the fact that the true constraints are known at time $k$ it is easy to calculate the bounded evolution of the system to time $k+1$ and therefore all possible states, $\mathcal{X}_{k+1}$. It is then obvious that for this limited subset of the state-space there might exist a better inner convex approximation of the nonlinear constraints than the global approximation $\mathcal{G}$. Hence, we would like to construct a convex polytope, $\mathcal{L}$, over the set $\mathcal{X}_{k+1}$ and constrain $\left(x_{k+1}, \tilde{u}_{k+1}\right)$ to this local approximation.

This procedure can of course be repeated for time step $k+2, k+3, \ldots, k+N-1$, generating a new local polytope for each $\left(x_{k+i}, \tilde{u}_{k+i}\right)$. A significant problem will however occur if one tries to prove recursive feasibility of this scheme. Since we always use the exact constraint for the first control input, this conflicts with our inner approximation which was used for future control input, when we shift the horizon in standard MPC stability and recursive feasibility proofs. If we use the full control authority in the next time instant, the state predictions arising from that set will move outside the predictions that were used in the previous time instant when predictions were based on an inner approximation of the control input at $k+1$. To account for this, a scheme based on both inner approximations of control inputs for actual control decisions, and outer approximations of control inputs to perform the propagation of states, will be used.

The local constraint approximations are constructed as inner convex approximations of the nonlinear constraints based on reachable sets.
Definition 1: At time $k$, the outer approximation of the $\mathrm{i}$ :th step reachable set $\mathcal{X}_{k+i}$ is recursively defined as

$$
\mathcal{X}_{k+i}=A \mathcal{X}_{k+i-1}+B \mathcal{O}_{k+i-1}
$$

where

$$
\mathcal{X}_{k}=\left\{x_{k}\right\}
$$

The set $\mathcal{O}_{k+i}$ is an outer polytopic approximation of the nonlinear control constraints in the reachable set $\mathcal{X}_{k+i}$, i.e.,

$$
\mathcal{O}_{k+i}=\left\{\tilde{u}_{k+i} \mid \omega_{l}^{k+i}\left(\mathcal{X}_{k+i}\right) \leq \tilde{u}_{k+i} \leq \omega_{u}^{k+i}\left(\mathcal{X}_{k+i}\right)\right\}
$$

where $\omega_{u}^{k+i}(\cdot)$ is a concave piecewise affine function such that

$$
\omega_{u}^{k+i}\left(\mathcal{X}_{k+1}\right) \geq \pi\left(\mathcal{X}_{k+1}, \bar{u}\right)
$$

and $\omega_{l}^{k+i}(\cdot)$ is a convex piecewise affine function that such that

$$
\pi\left(\mathcal{X}_{k+1}, \underline{u}\right) \geq \omega_{l}^{k+i}\left(\mathcal{X}_{k+1}\right)
$$

The initial outer approximation, $\mathcal{O}_{k}$, is the exact control constraints, i.e.,

$$
\mathcal{O}_{k}=\left\{u_{k} \mid \pi\left(x_{k}, \underline{u}\right) \leq \tilde{u}_{k} \leq \pi\left(x_{k}, \bar{u}\right)\right\}
$$

Assumption 3: For all reachable sets $\mathcal{X}_{k+i}$ for $i=1, \ldots, N-1$ the following hold

$$
\mathcal{X}_{k+i} \cap \mathcal{X} \neq \emptyset
$$

From the i:th step reachable set the local convex approximation, $\mathcal{I}_{i}^{k}, i$ step ahead at time $k$ can now be constructed as the polytope defined from the constraints

$$
\begin{gathered}
h_{l}^{k+i}\left(\mathcal{X}_{k+i} \cap \mathcal{X}\right) \leq \tilde{u}_{k+i} \leq h_{u}^{k+i}\left(\mathcal{X}_{k+i} \cap \mathcal{X}\right) \\
x_{k+i} \in \mathcal{X}_{k+i} \cap \mathcal{X}
\end{gathered}
$$

where $h_{u}^{k+i}(\cdot)$ is concave piecewise affine function such

$$
g_{u}\left(\mathcal{X}_{k+1}\right) \leq h_{u}^{k+i}\left(\mathcal{X}_{k+1}\right) \leq \pi\left(\mathcal{X}_{k+1}, \bar{u}\right)
$$

and $h_{l}^{k+i}(\cdot)$ is convex piecewise affine function such

$$
\pi\left(\mathcal{X}_{k+1}, \underline{u}\right) \leq h_{l}^{k+i}\left(\mathcal{X}_{k+1}\right) \leq g_{l}\left(\mathcal{X}_{k+1}\right)
$$

In other words, the local polytope, $\mathcal{I}_{i}^{k}$, shall be an inner approximation to the nonlinear constraints and on the subset $\mathcal{X}_{k+i}$ it shall hold that $\mathcal{G} \subseteq \mathcal{I}_{i}^{k}$, which can always be achieved. Figure 1 shows an example that illustrate the relationship between the local polytopes, the global polytope and the nonlinear constraints. Note that as for the global inner convex approximation this construction is non unique, in this paper we have used a tangent plane for the concave surfaces and a piecewise linear approximation of the convex surfaces (described further in the examples).

\section{B. MPC Receding Horizon Setup}

Now let us summarize the discussion above into our proposed MPC algorithm. 


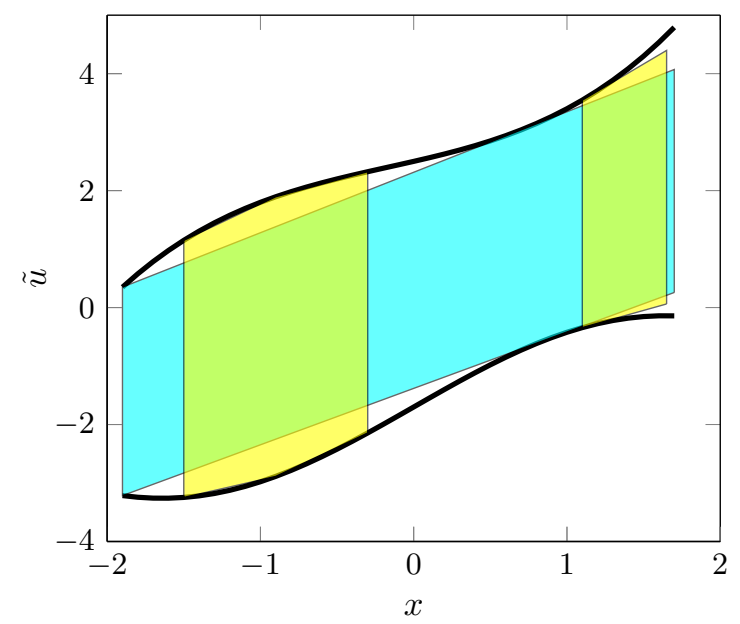

Fig. 1: Example showing the nonlinear constraints on $\tilde{u}$ as upper and lower bound, the global approximation, $\mathcal{G}$, in cyan (dark shaded) and two local approximations, $\mathcal{I}_{1}^{k}$, for different $x_{k}$ in yellow.

Algorithm 1: At each sample time $k$ solve the open loop optimal control problem

$$
\begin{aligned}
& \min _{u} \Psi\left(x_{k+N}\right)+\sum_{i=0}^{N-1} \ell\left(x_{k+i}, \tilde{u}_{k+i}\right) \\
& \text { s.t. } \\
& x_{k+i+1}= A x_{k+i}+B \tilde{u}_{k+i} \\
& \pi\left(x_{k}, \underline{u}\right) \leq \tilde{u}_{k} \leq \pi\left(x_{k}, \bar{u}\right) \\
&\left(x_{k+i}, \tilde{u}_{k+i}\right) \in \mathcal{I}_{i}^{k} \forall i=1, \ldots, N_{l} \\
&\left(x_{k+i}, \tilde{u}_{k+i}\right) \in \mathcal{G} \forall i=N_{l}+1, \ldots, N-1 \\
& x_{k+N} \in \mathcal{T}
\end{aligned}
$$

where the state and control are constrained to the local polytopes up to horizon $N_{l} \leq N-1$ and constrained to the global polytope for $N_{l}<i<N$. The introduction of the horizon $N_{l}$ is to highlight that, depending on the problem, there might not be any performance gain in using the local polytopes for the entire horizon, $N-1$, so instead using the fixed global inner approximation for the last part of the horizon.

For next time sample $k+1$ update the local approximations $\mathcal{I}_{i}^{k+1}$ as

$$
\mathcal{I}_{i}^{k+1}=\mathcal{I}_{i+1}^{k} \forall i=1, \ldots, N_{l}-1
$$

and construct a new set $\mathcal{I}_{N_{l}}^{k+1}$ from the procedure in Section II-A. The invariant set $\mathcal{T}$ in (13f) is calculated from the global convex approximation $\mathcal{G}$ as

$$
\mathcal{T}=\{x \mid f(x) \in \mathcal{T} \forall x \in \mathcal{T},(x, \kappa(x)) \in \mathcal{G}\}
$$

where $f(x)=A x+B \kappa(x)$.

We can now give the main result of this paper

Theorem 1: For any feasible initial state $x_{0}$, the MPC controller defined by Algorithm 1 remains feasible and stabilizes the system (10).
Here we outline only the proof of recursive feasibility, convergence of the proposed algorithm is not affected by the local approximations and standard proofs hold without any change, see e.g., [1].

Proof: Let us denote the set of states where (13) is feasible with $\mathcal{F}$. Assume that $x_{k} \in \mathcal{F}$ and (13) have the optimal solution sequence $\tilde{u}_{k: k+N-1}^{*}$.

We now claim that a feasible solution at time $k+1$ is to use $\hat{u}=\left[\tilde{u}_{k+1: k+N-1}^{*}, \kappa\left(x_{N}^{*}\right)\right]$ where $\kappa(x)$ is the local controller from Section I.

To see that this is a feasible solution we first note that since $x_{k+N} \in \mathcal{T}$ we can select $\hat{u}_{k+N}=\kappa\left(x_{N}^{*}\right)$ since this will ensure that $x_{k+N+1} \in \mathcal{T}$ and all constraints are satisfied at $k+N+1$. Also we note that since $\tilde{u}_{k+1}^{*} \in \mathcal{I}_{1}^{k} \subset \Pi$ this means that $\pi\left(x_{k+1}, \underline{u}\right) \leq \tilde{u}_{k+1}^{*} \leq \pi\left(x_{k+1}, \bar{u}\right)$ is feasible at time $k+1$.

Furthermore we have that all $\tilde{u}_{k+i}^{*}, i=2, \ldots, N_{l}$ are feasible at time $k+1$ since the local approximations are shifted one timestep (14). The control $\tilde{u}_{k+N_{l}+1}^{*} \in \mathcal{G}$ at time $k$ are also feasible at time $k+1$ since $\tilde{u}_{k+N_{l}+1}^{*} \in \mathcal{I}_{N_{l}}^{k+1} \supseteq \mathcal{G}$ at time $k+1$ and $\mathcal{I}_{N_{l}}^{k+1}$ is nonempty due to Assumption 3 . All other $\tilde{u}_{k+N_{l}+i}^{*} \in \mathcal{G}$ are trivially feasible.

\section{EXAMPLES}

In this section we present two examples to illustrate the properties of the proposed algorithm. In the first example we consider a fictitious nonlinear system whose purpose is to illustrate the generation and propagation of the local polytopes.

In the second example we consider the task of controlling a fighter aircraft which has nonlinear unstable dynamics. The purpose of this example is to illustrate the degree of suboptimality for the proposed method.

The implementation and simulation has been performed in MATLAB with YALMIP [14] and MPT [15].

\section{A. Illustrative Example}

Consider a nonlinear system given by

$$
\begin{aligned}
& \dot{x}=1.8 x+\left(0.2 x^{4}+0.875\right) u \\
& y=x
\end{aligned}
$$

with the constraints $-2 \leq x \leq 2$, and $-2 \leq u \leq 2$. Following the procedure in section I-A we obtain the feedback linearization control law

$$
u=\frac{1}{0.2 x^{4}+0.875}(\tilde{u}-1.8 x)
$$

and the resulting linear system is an integrator from $\tilde{u}$ to $y$.

The nonlinear feedback gives the following nonlinear control constraints on $\tilde{u}$

$$
-0.4 x^{4}+1.8 x-1.75 \leq \tilde{u} \leq 0.4 x^{4}+1.8 x+1.75
$$

shown in Figure 2.

The algorithm (13) is applied to the discrete-time version of the integrator system with sample time $0.4 s$. Using $N=5$ and $N_{l}=4$, i.e., we use the global polytope $\mathcal{G}$ only to calculate the terminal constraint set, $\mathcal{T}$, and the objective is 


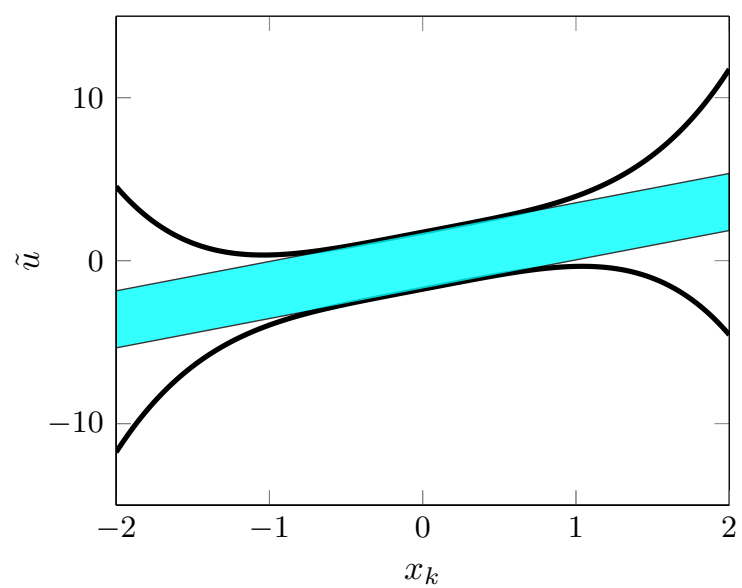

Fig. 2: Nonlinear constraints on $\tilde{u}$ due to feedback linearization and a global inner approximation $\mathcal{G}$. Note the massive loss of control authority at $x_{k}= \pm 2$.

to control the system to the origin. The local polytopes are calculated from the tangent line at the center point in the reachable set.

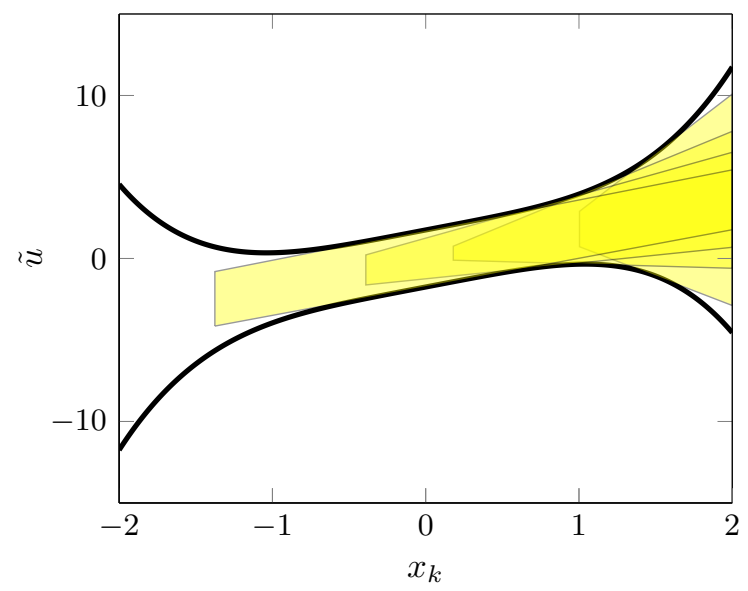

Fig. 3: Local polytopes $\mathcal{I}_{i}^{0}$ for $i=1, \ldots, 4$ at time $k=0$.

Starting in $x=1.9$ the generated local polytopes at time $k=0, \mathcal{I}_{i}^{0}$, are shown in Figure 3 . It clearly demonstrates the increased control signal ability compared to only using the global approximation.

At the next time step the first local approximation, $\mathcal{I}_{1}^{0}$ is discarded, all other polytopes are shifted one step, i.e. $\mathcal{I}_{1}^{1}=$ $\mathcal{I}_{2}^{0}, \mathcal{I}_{2}^{1}=\mathcal{I}_{3}^{0}$ etc. and a new one is generated at the end of the sequence, $\mathcal{I}_{4}^{1}$. Figure 4 shows how the local approximations look at time $k=2$, when $x \approx 0.6$. In the figure one can see that the polytope $\mathcal{I}_{3}^{0}$ from Figure 3 has been shifted and is now, at time $k=2$, the polytope $\mathcal{I}_{1}^{2}$. The same holds for $\mathcal{I}_{4}^{0}=\mathcal{I}_{2}^{2}$.

\section{B. Nonlinear Aircraft}

Using a simple model for the nonlinear dynamic equations of an aircrafts short period dynamics we obtain the following

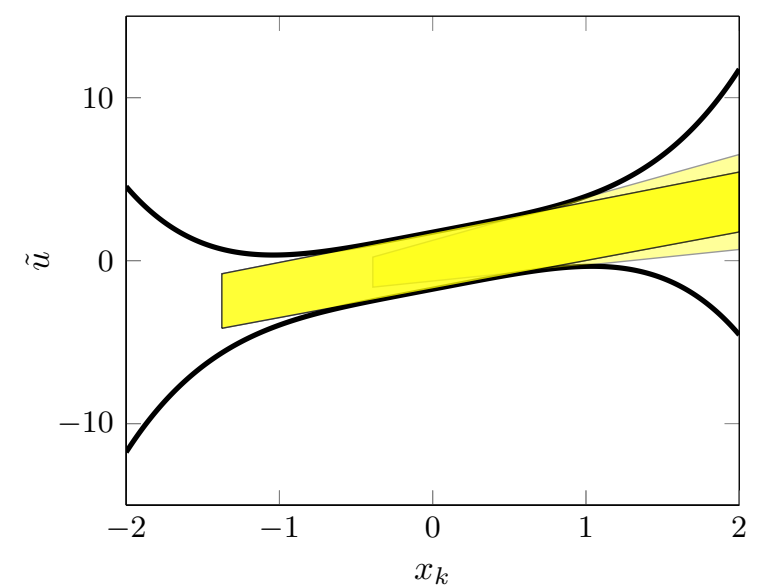

Fig. 4: Local polytopes $\mathcal{I}_{i}^{2}$ for $i=1, \ldots, 4$ at time $k=2$.

two state model

$$
\begin{aligned}
\dot{\alpha} & =-k_{1} \alpha+k_{2} q \\
\dot{q} & =k_{3} \alpha^{2}+k_{4} \alpha-k_{5} q+k_{6} \delta_{e} \\
y & =\alpha
\end{aligned}
$$

where $q$ is the angular velocity in pitch, $\alpha$ is angle between the aircraft $\mathrm{x}$-axis and the velocity vector. The input to the system is $\delta_{e}$ which is the elevator control surface deflection (for a complete description of aircraft dynamical equations see [16]). The constants $k_{1}, k_{2}, k_{4}, k_{5}$ and $k_{6}$ have been selected to correspond to the linearized dynamics of the ADMIRE model at Mach 0.6 and altitude $1000 \mathrm{~m}$, for details see, [17]. The constant $k_{3}$ is selected to make the $\alpha$-contribution to the moment equation (16b) approximate $15 \%$ larger at $\alpha=30^{\circ}$ than that of the linearized model.

The constraints on the system are basic control surface deflection limits $\left|\delta_{e}\right| \leq 25^{\circ}$ and a so called maneuver load limit on the angle of attack $-10^{\circ} \leq \alpha \leq 30^{\circ}$.

For the system (16) it is now easy to see that by selecting the nonlinear feedback as

$$
\delta_{e}=\tilde{u}-\frac{k_{3}}{k_{6}} \alpha^{2}
$$

the closed loop system from MPC control input, $\tilde{u}$, to the output, $\alpha$ will be linear

$$
\begin{aligned}
& \dot{\alpha}=-k_{1} \alpha+k_{2} q \\
& \dot{q}=k_{4} \alpha-k_{5} q+k_{6} \tilde{u}
\end{aligned}
$$

We can now formulate a MPC problem for the system (18) on the form (13) where we chose to use a cost function that is quadratic in $\tilde{u}$ since the goal is to end up in a standard MPC problem. In the cost function we used the tuning parameters

$$
Q=\left[\begin{array}{ll}
5 & 0 \\
0 & 1
\end{array}\right], \quad R=2
$$

and the sample time is $1 / 60$ second.

Note that the state constraints are still linear after the feedback linearization but the control constraints are now 
nonlinear and state dependent.

$$
-25^{\circ}+\frac{k_{3}}{k_{6}} \alpha_{k+i}^{2} \leq \tilde{u}_{k+i} \leq 25^{\circ}+\frac{k_{3}}{k_{6}} \alpha_{k+i}^{2}
$$

In this case the nonlinearities are mild and the lower bound is convex while the upper bound is concave. It is therefore quite easy to make a good inner convex polytope approximation, $\mathcal{G}$, of these constraints, see Figure 5.

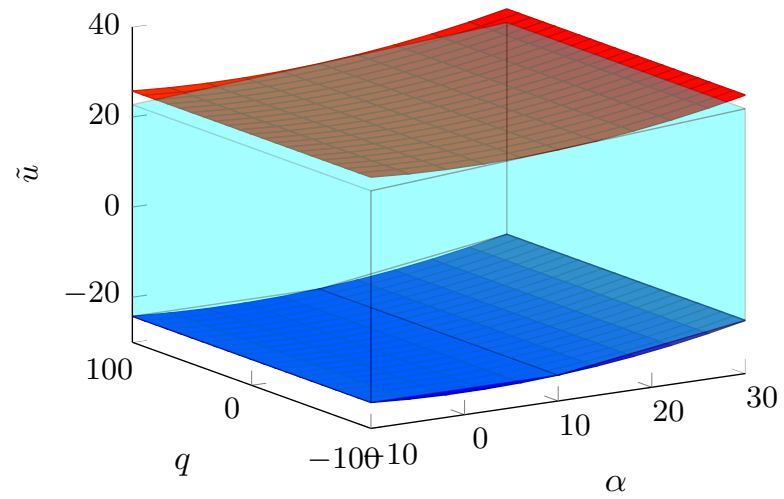

Fig. 5: Nonlinear control signal constraints (19) and a global inner convex polytope approximation, $\mathcal{G}$

If we compare the proposed algorithm (13), with $N_{l}=1$ and $N_{l}=10$, with the global nonlinear branch and bound solver in YALMIP [14] that uses the exact control constraints (11), we obtain a measure of the performance loss, the suboptimality of our algorithm. We have compared the open loop optimal cost of the two algorithms for a set of different initial conditions which are steady state points distributed over the complete $\alpha$ range. In Table I we show the relative error

$$
\eta=\frac{\left|J^{*}-\hat{J}\right|}{\left|J^{*}\right|}
$$

between the optimal cost of the proposed algorithm, $\hat{J}$, and the branch and bound solver, $J^{*}$.

TABLE I: Relative error, $\eta$, between proposed algorithm and global solver for different initial conditions and number of local polytope approximations

\begin{tabular}{ccccc}
\hline \hline$x_{0}$ & $\left(\begin{array}{l}-14.0 \\
-26.5\end{array}\right)$ & $\left(\begin{array}{l}-5.0 \\
-9.5\end{array}\right)$ & $\left(\begin{array}{l}15.0 \\
28.3\end{array}\right)$ & $\left(\begin{array}{l}28.0 \\
52.9\end{array}\right)$ \\
\hline$N_{l}=1$ & $0.4 \cdot 10^{-3}$ & $0.0 \cdot 10^{-3}$ & $0.8 \cdot 10^{-3}$ & $24.6 \cdot 10^{-3}$ \\
$N_{l}=10$ & $0.0 \cdot 10^{-3}$ & $0.0 \cdot 10^{-3}$ & $0.0 \cdot 10^{-3}$ & $10.8 \cdot 10^{-3}$ \\
\hline \hline
\end{tabular}

From the results in Table I we can see that the maximum suboptimality obtained is approximately $2.4 \%$ and that in this example, when there are only small nonlinearities, there is no significant gain in increasing the number of local polytopes, i.e., the important property is that we can use the full control authority for the current control input.

\section{CONCLUSIONS}

In this paper we have demonstrated a novel method of model predictive control for nonlinear systems based on feedback linearization and local convex approximations of the control constraints.

We have shown recursive feasibility and convergence to the origin and that the loss of optimality can be small with reasonable simple computational efforts.

In this paper have we disregarded the fact that the feedback linearization control law is derived in continuous time while the model predictive controller works in discrete time. Hence we asume there is a inner linearizing feedback loop.

Acknowledgement: This work has been done as a cooperation between Linköping University and Saab Aeronautics and is funded by the Swedish Governmental Agency for Innovation Systems (VINNOVA) and Center for industrial information technology (CENIIT).

\section{REFERENCES}

[1] D. Mayne, J. B. Rawlings, C. V. Rao, and P. Scokaert, "Constrained model predictive control: Stability and optimality," Automatica, vol. 36, no. 6, pp. 789-814, Jun. 2000.

[2] K. R. Muske and J. B. Rawlings, "Model predictive control with linear models," AIChE Journal, vol. 39, no. 2, pp. 262-287, Feb. 1993.

[3] F. Blanchini, "Set invariance in control," Automatica, vol. 35, pp. 1747 - 1767, 1999.

[4] S. Boyd and L. Vandenberghe, Convex Optimization, 1st ed. Cambridge University Press, 2004.

[5] M. Cannon, "Efficient nonlinear model predictive control algorithms," Annual Reviews in Control, vol. 28, no. 2, pp. 229-237, Jan. 2004.

[6] L. Del Re, J. Chapuis, and V. Nevistik, "Predictive Control with Embedded Feedback Linearization for Bilinear Plants with Input Constraints," in Proceedings of the 32th IEEE Conference on Decision and Control (CDC), 1993, pp. 2984-2989.

[7] H. K. Khalil, Nonlinear Systems third edition. Prentice Hall, 2002.

[8] J. A. Primbs and V. Nevistic, "MPC Extensions to Feedback Linearizable Systems," in Proceedings of the 1997 American Control Conference, vol. 0, no. June, 1997, pp. 2073-2077.

[9] J. Deng, V. M. Becerra, and R. Stobart, "Input Constraints Handling in an MPC/Feedback Linearization Scheme," International Journal of Applied Mathematics and Computer Science, vol. 19, no. 2, pp. 219232, Jun. 2009.

[10] K. Margellos and J. Lygeros, "A simulation based MPC technique for feedback linearizable systems with input constraints," in 49th IEEE Conference on Decision and Control (CDC). Ieee, Dec. 2010, pp. 7539-7544.

[11] M. V. Kothare, V. Nevistic, and M. Morari, "Robust Constrained Model Predictive Control for nonlinear Systems: A Comperative Study," in Proceedings of the 34th IEEE Conference on Decision and Control (CDC), no. December, 1995, pp. 2884-2885.

[12] V. Nevistic and L. Del Re, "Feasible Suboptimal Model Predictive Control for Linear Plants with State Dependent Constraints," in Proceedings of the 1994 American Control Conference, vol. 2862, no. 1, 1994.

[13] M. J. Kurtz and M. A. Henson, "Feedback linearizing control of discrete-time nonlinear systems with input constraints," International Journal of Control, vol. 70:4, pp. 603-616, 2010.

[14] J. Löfberg, "YALMIP: A Toolbox for Modeling and Optimization in MATLAB," in Proceedings of the CACSD Conference, Taipei, Taiwan, 2004. [Online]. Available: http://users.isy.liu.se/johanl/yalmip/

[15] M. Kvasnica, P. Grieder, and M. Baotic, "Multi-Parametric Toolbox (MPT)," Hybrid Systems: Computation and Control, pp. 121 - 124, 2004.

[16] B. L. Stevens and F. L. Lewis, Aircraft Control and Simulation, 2nd ed. John Wiley \& Sons, 2003.

[17] L. Forssell and U. Nilsson, "ADMIRE The Aero-Data Model In a Research Environment Version 4.0, Model Description," Swedish Defence Research Agency, Tech. Rep. December, 2005. 